\title{
EXPONENTIALLY LONG TIMES TO EQUIPARTITION IN THE THERMODYNAMIC LIMIT
}

\author{
LUISA BERCHIALLA, ANTONIO GIORGILLI ${ }^{\dagger}$ and SIMONE PALEARI \\ Università di Milano Bicocca, Dipartimento di Matematica e Applicazioni, \\ Via degli Arcimboldi 8, 20126 - Milano, Italy.
}

\begin{abstract}
We investigate with numerical methods the scaling of the relaxation time to equipartion in the celebrated Fermi-Pasta-Ulam model. Our numerical results strongly suggest that the time increases exponentially with an inverse power of the specific energy. Such a scaling appears to remain valid in the thermodynamic limit.
\end{abstract}

PACS numbers: 05.45.--a, 63.20.Ry, 63.10.+a

Keywords: Nonlinear chain, FPU model, Thermodynamic limit.

\section{Introduction}

The FPU paradox, originated by the celebrated report of Fermi, Pasta and Ulam ${ }^{[12]}$, has been the subject of many studies in the past decades. The facts are well known. One is interested in the evolution towards equipartition of energy in a chain of non-linearly coupled oscillators with identical masses if the energy is initially given to one or a few modes. For small energies, one observes instead an unexpected behaviour: the energy remains concentrated only on a few modes. Further investigation has shown that the sharing of energy, if it happens, takes a very long time that becomes unobservable even with the most powerful computers. The problem is to explain this phenomenon, and to prove or disprove its persistence in the thermodynamic limit.

Two different conjectures have been raised at the very beginning of this long story. On the one hand, Izrailev and Chirikov tried to interpret the FPU phenomenon as due to the existence of an energy threshold $E_{c}$ below which equipartition can not occur ${ }^{[20]}$. However, they conjectured that the FPU phenomenon could not persist when the number of particles grows very large, due to the presence of many resonances among the frequencies of the normal modes: the energy threshold $E_{c}$ should vanish in the thermodynamic limit. In a paper by Shepelyansky ${ }^{[27]}$ it is argued that the conjecture of Izrailev and Chirikov was finally proved. On the other hand, Bocchieri, Scotti, Bearzi

$\dagger$ Corresponding author. Ph. n. xx39-02-64487761; Fax n. xx39-02-6448 7705; e-mail: antonio@matapp. unimib.it. 
and Loinger conjectured the existence of threshold $\varepsilon_{c}$ in specific energy (or energy per particle $\varepsilon=E / N)$ that is independent of the number $N$ of particles ${ }^{[3]}$. The relevance of the latter conjecture was particularly stressed by Cercignani, Galgani and Scotti ([14], [15], [7]; see also [4]).

Several papers have been devoted to the problem of identifying such thresholds, and in particular to investigate their persistence for large $N$ : see, e.g., [5], [21], [23], $[24],[25],[22],[6]$. However, it seems appropriate to say that a definite conclusion has not been reached till now.

More recently a new interpretation has been proposed, based on the Nekhoroshev's theory on exponential stability: equipartition should always occur, but when the specific energy is small enough it could take an exponentially long time that might even overcome the lifetime of a physical system ${ }^{[16]}$. This idea was apparently first proposed in a paper by Fucito et al. ${ }^{[13]}$. It is natural to identify the threshold conjectured by Bocchieri, Scotti, Bearzi and Loinger with the energy below which the relaxation time to equipartition begins to increase as $\exp \left(\varepsilon^{-a}\right)$, with some positive $a$.

The time scale to equipartition has been investigated by some authors. Pettini and Landolfi suggest that an exponential law with parameters independent of the number of particles should hold ${ }^{[26]}$. De Luca, Lichtenberg and Lieberman make a careful study of the role of resonances in driving the sharing of energy among modes ${ }^{[8]}$. De Luca, Lichtenberg and Ruffo in a series of papers concentrate their attention on the range of energy where the sharing of energy actually occurs ${ }^{[9][10][11]}$, and suggest that an appropriate scaling law for the relaxation time is a power law $\varepsilon^{-a}$.

The considerations of the present paper are based on the work [11] of De Luca, Lichtenberg and Ruffo and on the recent paper [2] by Berchialla, Galgani and Giorgilli. In [2] a numerical evidence of the existence of two well separated time scales has been produced. The first time scale corresponds to a fast flow of energy from the first initially excited mode to the next few low-frequency modes. This creates what has been called a natural packet, which persists for a long time. The second time scale corresponds to the destruction of the natural packet, due to a further flowing of energy towards the highest modes. It is this second time scale which is of particular interest, since it may be naturally identified with the relaxation time to equipartition. However, no clear indication concerning the scaling of the latter time with the specific energy is given (see sect. 3 for more details). In [11] De Luca, Lichtenberg and Ruffo give a theoretical and numerical estimate of the relaxation time to equipartition, suggesting a scaling law $T \sim \varepsilon^{-3}$ (see formula (17) in that paper).

Now, a direct comparison of the results in [2] and [11] is impossible, due to different choices of the model, the initial conditions, and the parameters. Thus, we performed a numerical investigation using the same model, class of initial conditions and parameter as in [11]. As a result of our investigations it turns out that the power law $T \sim \varepsilon^{-3}$ appears somehow to be an artifact due to the choice of a too restricted energy range, and that extending the calculations to a wider range leads to a different conclusion. Indeed, our calculations indicate that the relaxation time to equipartition may well increase as 
$T \sim \exp \left(\varepsilon^{-1 / 4}\right)$. It might be useful to recall here a general property which helps to understand how it can occur that the data appear to fit a power law in some region, and to better fit an exponential law in a larger one. The point is that a typical result of perturbation theory is indeed that some relevant quantity remains almost invariant for a time of order $\varepsilon^{-r}$ for some $r \geq 1$. The exponential time typical of Nekhoroshev's theory is then the outcome of an optimal choice of $r$ as a function of $\varepsilon$, i.e., $r \sim \varepsilon^{-a}$ for some $a$ (see, e.g., [18] for an informal exposition and [19] for a quantitative application).

\section{The model}

The FPU $\beta$-model that we are considering is a one-dimensional chain of $N+2$ particles with fixed ends, as described by the Hamiltonian

$$
H(x, y)=\frac{1}{2} \sum_{j=1}^{N} y_{j}^{2}+\frac{1}{2} \sum_{j=0}^{N}\left(x_{j+1}-x_{j}\right)^{2}+\frac{\beta}{4} \sum_{j=0}^{N}\left(x_{j+1}-x_{j}\right)^{4} .
$$

Here, $x_{1}, \ldots, x_{N}$ are the displacements with respect to the equilibrium positions (that obviously exist), and $x_{0}=x_{N+1}=0$ are the fixed ends. The normal modes are introduced via the canonical transformation

$$
x_{j}=\sqrt{\frac{2}{N+1}} \sum_{k=1}^{N} q_{k} \sin \frac{j k \pi}{N+1}, \quad y_{j}=\sqrt{\frac{2}{N+1}} \sum_{k=1}^{N} p_{k} \sin \frac{j k \pi}{N+1},
$$

$\left(q_{k}, p_{k}\right)$ being the new coordinates and momenta. The quadratic part of the Hamiltonian in the normal coordinates is given the form

$$
H_{2}=\sum_{j=1}^{N} E_{j}, \quad E_{j}=\frac{1}{2}\left(p_{j}^{2}+\omega_{j}^{2} q_{j}^{2}\right)
$$

with harmonic frequencies

$$
\omega_{j}=2 \sin \frac{j \pi}{2(N+1)}
$$

We investigate numerically the model with $\beta=1 / 10$, and with $N$ typically ranging from 255 to 1023; in a few cases $N$ has been increased up to 32767 . The integration was performed using a leap-frog method, with typical time step 0.05. Except when explicitly indicated, the initial conditions were always taken as in [11]. I.e., the total energy $E$ is equally distributed among the modes between $(N+1) / 64$ and $5(N+1) / 64$. 

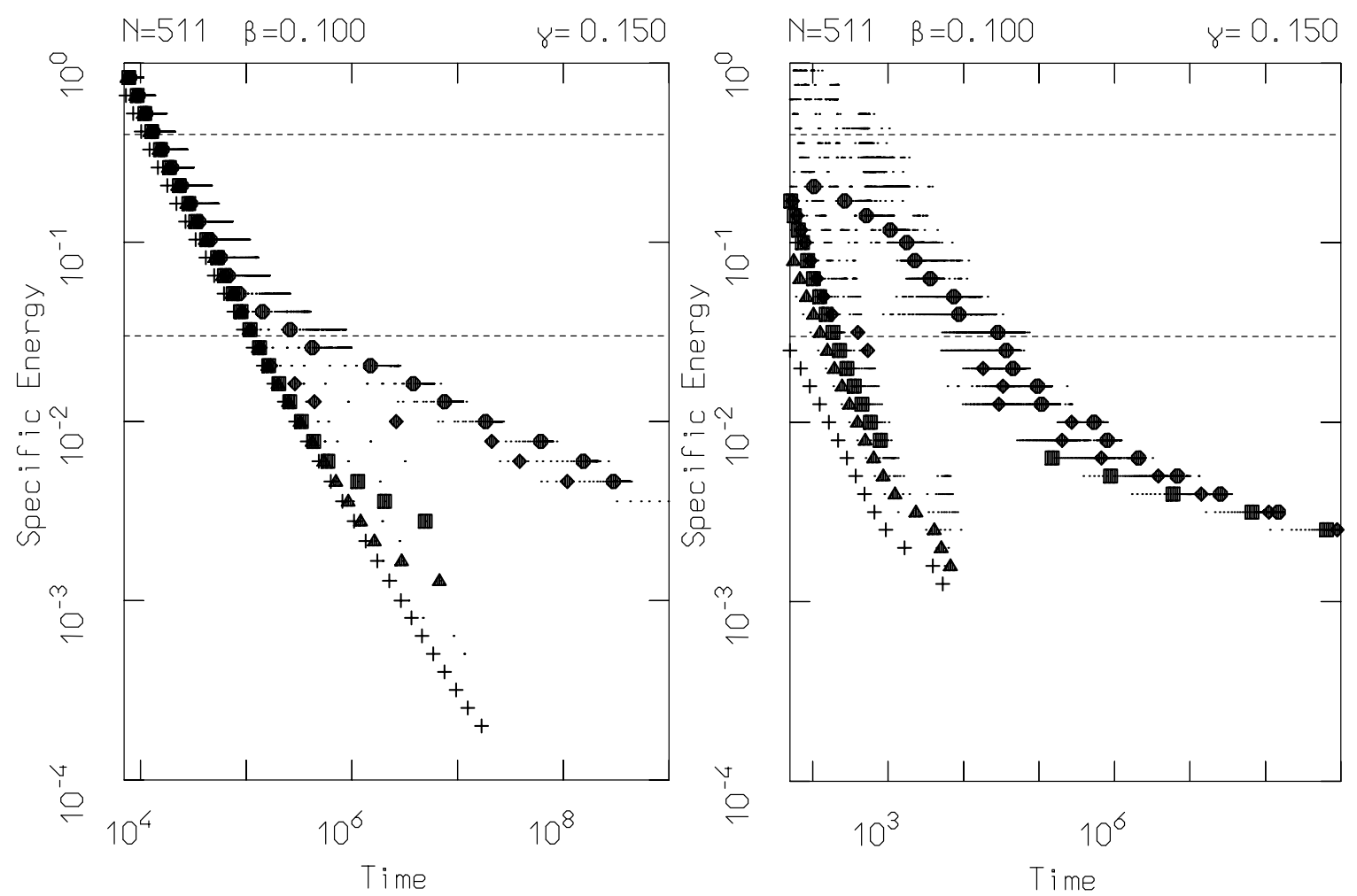

Figure 1. The critical time $t_{s}^{R}$ (abscissas) corresponding to a specific energy $\varepsilon$ (ordinates) for all packets. Number of particles: 511. Left figure: initial conditions with the whole energy on the lowest frequency mode; crosses: $s=1$, triangles: $s=4$, squares: $s=8$, diamonds: $s=16$, circles: $s=32$. Right figure: initial condition with energy equally distributed among the modes 8-40; crosses: $s=35$, triangles: $s=37$, squares: $s=63$, diamonds: $s=127$, circles: $s=255$. The dots correspond to the other values of $s$.

\section{The phenomenon of the natural packets}

Our starting point is the formation of natural packets and their subsequent destruction, as observed in [2]. We consider the quantities

$$
\mathcal{E}_{1}=E_{1}, \quad \mathcal{E}_{2}=E_{1}+E_{2}, \ldots, \quad \mathcal{E}_{s}=E_{1}+\ldots+E_{s}, \ldots
$$

i.e., the total harmonic energy of the packet of the first $s$ modes, $s=1, \ldots, n$. Assume that at time $t=0$ we have $\mathcal{E}_{s}=E$ for a given $s$. If the system evolves towards equipartition we expect $\overline{\mathcal{E}}_{s}(t) \rightarrow s E / N$ when time increases to infinity (over line denotes the time average), i.e., the energy $\mathcal{E}_{s}$ is decreased by $(N-s) E / N$. The critical time $t_{s}^{R}$ is the first instant at which $\overline{\mathcal{E}}_{s}(t)$ has lost a fraction $\gamma$ of the latter quantity, with $0<\gamma<1$. The numerical data for $\left(t_{s}^{R}, \varepsilon\right)$ are plotted in fig. 1 , for $N=511$ and $\gamma=0.15$. The dashed lines delimit the interval of specific energy $0.03 \leq \varepsilon \leq 0.4$ considered in [11].

Let us first consider the left side of fig. 1 . The whole energy is initially on the lowest 
frequency mode, as in the classical experiment of Fermi, Pasta and Ulam. Thus, we have $\mathcal{E}_{s}=E$ for $s=1, \ldots, N$. For a constant value of the initial energy, i.e., moving on a horizontal line, the points corresponding to increasing values of $s$ are aligned from left to right. The relevant information here is that the energy flows quite rapidly from the initially excited modes to the next ones, but for some value of $s$ the flow stops, and a much longer time is needed for the quantity $\overline{\mathcal{E}_{s}}(t)$ to fall below the wanted threshold. In this case we say that the first $s$ modes form a natural packet that persists for a long time. The figure also shows that if we let the value of the energy decrease, then the length of the natural packet decreases, too, and the time needed for the energy to flow to higher modes significantly increases. This is the phenomenon described in [2].

The comparison with [11] is illustrated by the right side of fig. 1 . The energy is equally distributed among the modes $8-40$, as described at the end of sect. 2. Thus, we have initially $\mathcal{E}_{s}=0$ for $1 \leq s<8$ and $\mathcal{E}_{s}=E$ for $s \geq 40$. For $8 \leq s \leq 40$ the energy $\mathcal{E}_{s}$ of the packets increases linearly from 0 to $E$. There are initially a number of packets with energy less than the wanted threshold, so that the natural packets of low frequency modes are not well defined. However, this is actually harmless. Indeed, the right side of fig. 2 strongly supports the conjecture that after a short time the system reaches a metastable state in which a splitting of the system in two separated components seems to occur. The situation looks similar to the one described in [16] for a FPU system with alternating masses, where there is a natural splitting of the system in the populations of acoustic (low frequency) and optical (high frequency) modes. Here, it is not evident a priori how to separate the frequencies of the modes. In fact, the system itself finds its natural splitting, depending on the specific energy $\varepsilon$. We have checked that the same phenomenon occurs for $N$ ranging from 31 to 1023. Moreover, the qualitative aspects of the figure do not change significantly when the parameter $\gamma$ varies between 0.05 and 0.95 . We emphasize that although no data points appear for the interval $10^{-4}<\varepsilon<10^{-3}$ we actually did the calculation: the critical time in this range overcomes the limits of our numerical experiment.

Now, the problem is to make a quantitative estimate of the relaxation time to equipartition, i.e., the second and longer relaxation time that shows up in the figure. According to the general theory developed in [1] the rate of exchange of energy between the two populations becomes exponentially small with a scaling law of the form $\exp \left(-\mu^{-a}\right)$, where $\mu$ is the size of the perturbation and $a$ is some positive number less than 1. In particular, it is shown that resonances may play an active role in creating metastable states, possibly with an internal chaotic dynamics. In such a case, theoretical considerations suggest that typical values for $a$ could be $1 / 2$ or $1 / 4$. Although, strictly speaking, the theoretical results do no apply in a straightforward manner to the FPU model, we may conjecture that the existence of strong resonances among the low frequencies may create such a kind of metastable state. Since in the case of the FPU system the natural perturbation parameter is the amplitude of the oscillation, i.e., $\varepsilon^{1 / 2}$, we conjecture that an exponential law $t_{\text {eff }} \sim \exp \left(\varepsilon^{-1 / 4}\right)$ may be appropriate for our case. This is the conjecture that we want to test with a numerical experiment. 


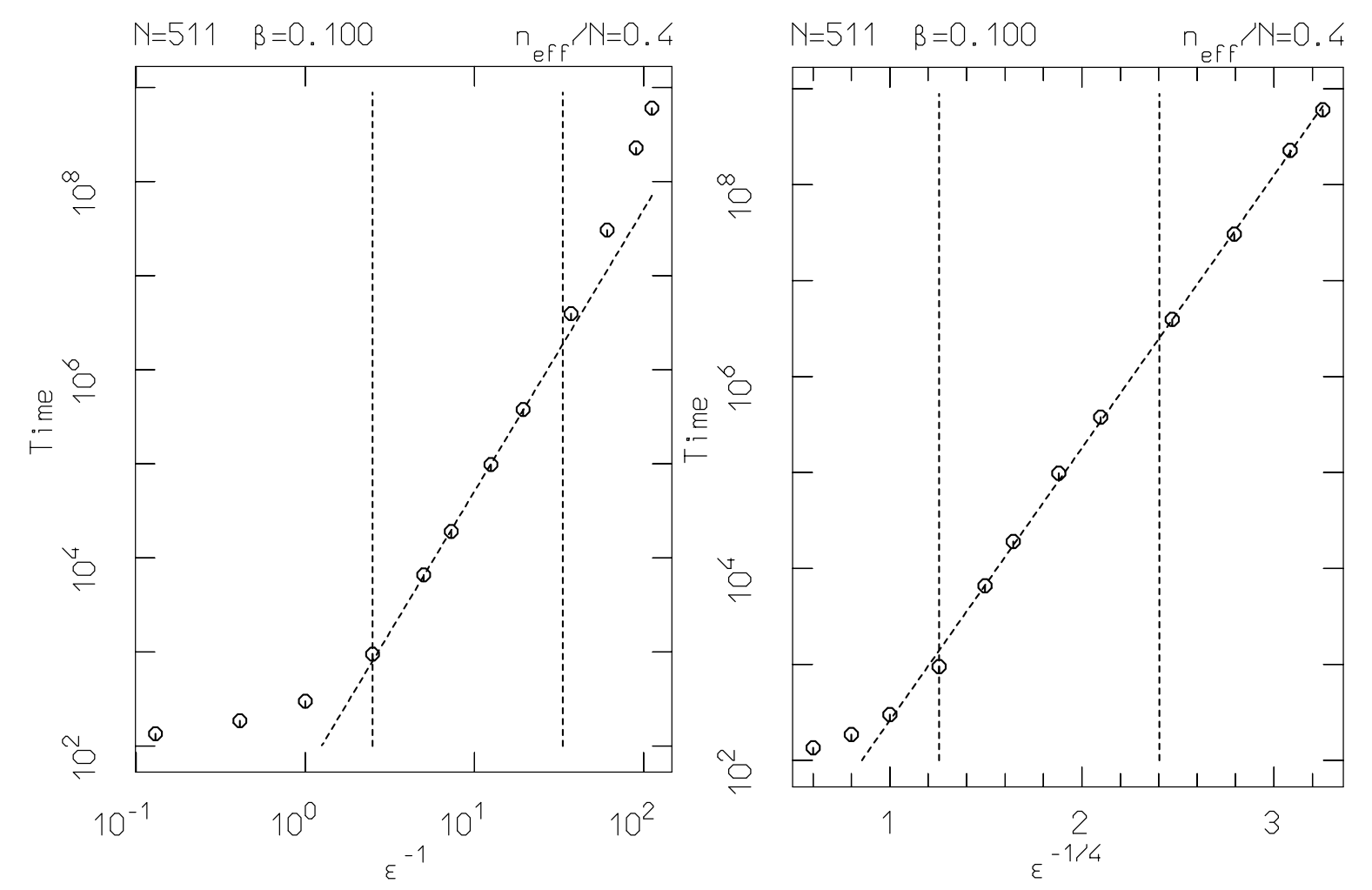

Figure 2. The time $t_{\text {eff }}$ needed to reach $n_{\text {eff }} / N=0.4$ vs. $\varepsilon^{-1}$, in $\log -\log$ scale (left side) and vs. $\varepsilon^{-1 / 4}$ in semi-log scale (right side). Left figure: the straight line is the best fit using the data in the interval $0.03 \leq \varepsilon \leq 0.4$, which has been considered in [11]. The slope is 2.99 , which is consistent with the predictions in that paper. Right figure: the straight line is the best fit using the data for $\varepsilon \leq 1$.

\section{Numerical estimate of the relaxation time to equipartition}

In order to investigate how the system relaxes to equipartition we use the same parameter $n_{\text {eff }}$ and the same method as in [11]. We recall the relevant definitions. The harmonic energies $E_{j}$ defined by (2) are renormalized as $e_{j}=E_{j} / \sum_{k} E_{k}$. The spectral entropy is then defined as $S=-\sum_{j} e_{j} \ln e_{j}$. This is a quantity ranging from 0 (when a single mode owns all the energy) to $\ln N$ when the energy is equally distributed among the modes. As suggested in [8], [9] and [10], a better quantity to be calculated is $n_{\text {eff }}=e^{S}$, which may be interpreted as the effective number of modes that share the energy.

In this paper we calculate the average of $n_{\text {eff }} / N$ over 25 different orbits at the same energy, with the same distribution of energy among the modes, and with a random choice of the initial phases. The value $n_{\mathrm{eff}}(t) / N$ is observed to increase from 0 to a maximal value around 0.66. The observed behaviour is the same as in fig. 3 of [11], so we omit the figure. In order to give a quantitative evaluation of the relaxation time to equipartition we look for a time $t_{\text {eff }}$ at which $n_{\text {eff }}(t) / N$ reaches a fixed value, that we take 0.4 , as in [11]. Looking at figure 1 it is quite evident that for specific energy below 


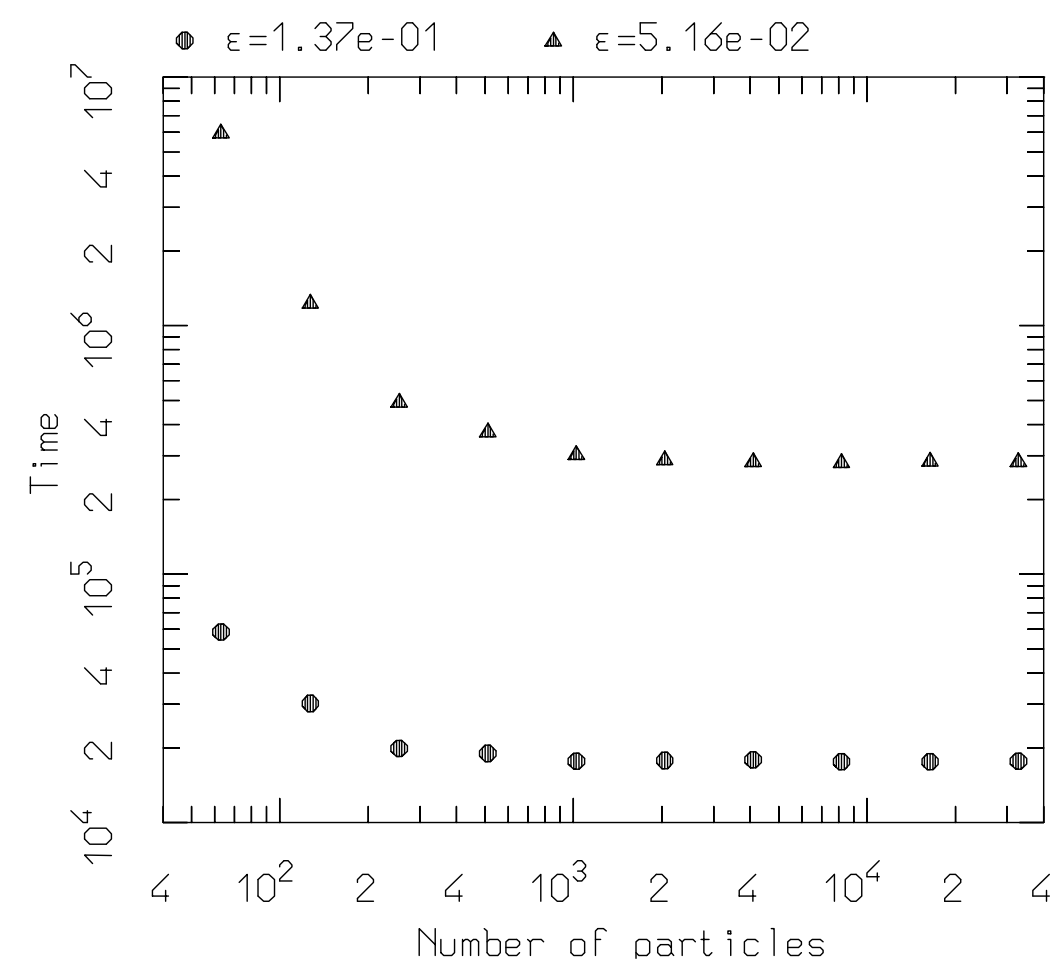

Figure 3. Dependence of the relaxation time to equipartition on the number $N$ of particles, for two different values of the specific energy $\varepsilon$, namely $\varepsilon=0.137$ (circles) and $\varepsilon=0.0516$ (triangles). The figure shows that the time tends to a constant value for large $N$, thus supporting the conjecture that the exponential law remains valid in the thermodynamic limit.

about $5 \times 10^{-3}$ the calculation will be in the best case impractical. Thus, we performed our investigation in the interval $8.9 \times 10^{-3} \leq \varepsilon \leq 7.7$. The results are illustrated in fig. 2, using different scales.

The scaling law $t_{\text {eff }} \sim \varepsilon^{-3}$ predicted in [11] is checked in the left part of the figure. The prediction is confirmed by the experimental data in the range $0.03 \leq \varepsilon \leq 0.4$; the interval is marked by the vertical dashed lines. However, by extending the specific energy range of the investigations the cubic scaling law seems not to be appropriate, since it fails to fit the experimental data.

The right side of fig. 2 tests the conjecture $t_{\mathrm{eff}} \sim \exp \left(\varepsilon^{-1 / 4}\right)$ made at the end of sect. 3. The comparison with the experimental data shows that the exponential fitting is definitely better than the cubic one. The deviation from the exponential law of the data for $\varepsilon>1$ is not surprising, because the data of fig. 1 show that no splitting of the system occurs for such an high energy. It would be interesting to better investigate the exponential law by further decreasing the specific energy, but the exponential increase of the computational time makes such a calculation actually impossible.

We come now to discuss how the time $t_{\text {eff }}$ depends on the number $N$ of particles. As a matter of fact, for $N$ ranging from 63 to 1023 our data confirm the exponential 
dependence of the relaxation time to equipartition on $\varepsilon^{-1 / 4}$. However, for a fixed value $\varepsilon$ the time $t_{\text {eff }}$ is observed to actually depend on $N$, and in particular to significantly decrease for low values of $N$. Therefore, we checked such a dependence by letting $N$ to increase up to 32767 . The results for two fixed values of $\varepsilon$ are illustrated in fig. 3 . It is immediate to observe that the time is definitely stabilized for $N \geq 1023$, indicating that the exponential law could survive in the thermodynamic limit.

\section{References}

[1] Benettin, G., Galgani, L. and Giorgilli, A.: Realization of holonomic constraints and freezing of high frequency degrees of freedom in the light of classical perturbation theory, part II, Comm. Math. Phys, 121, 557-601 (1989).

[2] Berchialla, L., Galgani, L. and Giorgilli, A.: Localization of energy in FPU chains, DCDS-B, to appear. (available at http://www.matapp.unimib.it/ ^antonio/ricerca/pubblicazioni.htm)

[3] Bocchieri, P., Scotti, A., Bearzi, B. and Loinger, A.: Anharmonic chain with Lennard-Jones interaction, Phys. Rev. A 2, 2013-2019 (1970).

[4] Carati, A., Galgani, L., Ponno, A. and Giorgilli, A.: The Fermi-Pasta-Ulam problem, Nuovo Cimento B 117, 1017-1026 (2002).

[5] Carotta, M.C., Ferrario, C., Lo Vecchio, G., Carazza, B. and Galgani, L.: New phenomenon in the stochastic transition of coupled oscillators, Phys. Rev A 17, 786 (1978).

[6] Casetti, L., Cerruti-Sola, M., Pettini, M. and Cohen, E.G.D.: The Fermi-PastaUlam problem revisited: Stochasticity thresholds in nonlinear Hamiltonian systems, Phys. Rev. E 55, 6566-6574 (1997).

[7] Cercignani, C., Galgani, L. and Scotti, A.: Phys. Lett. A, 38, 403 (1972).

[8] De Luca, J., Lichtenberg, A.J. and Liebermann, M.A.: Time scale to ergodicity in the Fermi-Pasta-Ulam system, Chaos 5, 283-297 (1995).

[9] De Luca, J., Lichtenberg, A.J. and Ruffo, S.: Energy transition and time scale to equipartition in the Fermi-Pasta-Ulam oscillator chain, Phys. Rev. E 51, 2877-2884 (1995).

[10] De Luca, J., Lichtenberg, A.J. and Ruffo, S.: Universal evolution to equipartition in oscillator chains, Phys. Rev E 54, 2329-2333 (1996).

[11] De Luca, J., Lichtenberg, A.J. and Ruffo, S.: Finite time to equipartition in the thermodynamic limit, Phys. Rev E 60, 3781-3786 (1999).

[12] Fermi, E., Pasta, J. and Ulam, S.: Studies of nonlinear problems, Los Alamos document LA-1940 (1955).

[13] Fucito, F., Marchesoni, F., Marinari, E., Parisi, G., Peliti, L., Ruffo, S., Vulpiani, A.: Approach to equilibrium in a chain of nonlinear oscillators, J. Physique 43, 707-713 (1982).

[14] Galgani, L. and Scotti, A.: Phys. Rev. Lett. 28, 1173 (1972). 
[15] Galgani, L. and Scotti, A.: Riv. Nuovo Cimento 2, 189 (1972).

[16] Galgani, L., Giorgilli, A., Martinoli, A. and Vanzini, S.: On the problem of energy equipartition for large systems of the Fermi-Pasta-Ulam type: analytical and numerical estimates, Physica D 59, 334-348 (1992).

[17] Giorgilli, A.: Rigorous results on the power expansions for the integrals of a Hamiltonian system near an elliptic equilibrium point, Ann. Ist. H. Poincaré, 48, n .4, 423-439 (1988).

[18] Giorgilli, A.: New insights on the stability problem from recent results in classical perturbation theory, in Les méthodes modernes de la mécanique célèste. Benest, D. and Froeschlé, C., eds., Ed. Frontières (1990).

[19] Giorgilli, A. and Skokos, Ch.: On the stability of the Trojan asteroids, Astron. Astroph. 317, 254-261 (1997).

[20] Izrailev, F.M. and Chirikov, B.V.: Stochasticity of the simplest dynamical model with divided phase space Dokl. Akad. Nauk. SSSR 166, 57 (1966); Sov. Phys. Dokl. 11, 30 (1966).

[21] Kantz, H.: Vanishing stability thresholds in the thermodynamic limit of nonintegrable conservative systems, Physica D 39, 322-335 (1989).

[22] Kantz, H., Livi, R. and Ruffo, S.: Equipartition thresholds in chains of anharmonic oscillators, J. Stat. Phys. 76, 627-643 (1994).

[23] Livi, R., Pettini, M., Ruffo, S., Sparpaglione, M. and Vulpiani, A.: Relaxation to different stationary states in the Fermi-Pasta-Ulam model, Phys. Rev A 28, 3544-3552 (1983).

[24] Livi, R., Pettini, M., Ruffo, S., Sparpaglione, M. and Vulpiani, A.: Equipartition threshold in nonlinear large Hamiltonian systems: the Fermi-Pasta-Ulam model, Phys. Rev. A 31, 1039-1045 (1985).

[25] Livi, R., Pettini, M., Ruffo, S. and Vulpiani, A.: Chaotic behaviour in nonlinear Hamiltonian systems and equilibrium statistical mechanics, J. Stat. Phys. 48, 539-559 (1987).

[26] Pettini, M. and Landolfi, M.: Relaxation properties and ergodicity breaking in nonlinear Hamiltonian dynamics, Phys. Rev A 41, 768-783 (1990).

[27] Shepelyansky, D.L.: Low-energy chaos in the Fermi-Pasta-Ulam problem, Nonlinearity, 10, 1331-1338 (1997). 